Method STAK is a 12 week, activity programme including activity diary, street dance DVD, circuit training and, for children at or above the $91^{\text {st }}$ centile weight for height, motivational interviewing and goal setting. STAK was evaluated in a cluster-randomised trial in 24 schools. Children aged 9 to 11 were screened for overweight, low exercise self-efficacy or asthma. Twelve schools were randomised to receive the STAK intervention and 12 to control. BMI, waist circumference and exercise self-efficacy were assessed at baseline and post intervention ( 4 months).

Results Of the 2479 children screened, 1065 children (43\%) met the study inclusion criteria. Parents of 424 (40\%) children consented to their child's participation with 4 months follow-up data available for 392 (92\%). The groups were well matched at baseline. After controlling for baseline values and time between testing, children in the intervention group had higher total self-efficacy at 4 month follow-up. In the group of children who were overweight at baseline $\left(\Rightarrow 91^{\text {st }}\right.$ centile), those in the STAK intervention group had smaller waist circumference and lower BMI at 4 month follow-up.

Conclusion Preliminary analysis suggests that a targeted activity intervention has benefits for children at risk of obesity. Future analyses will explore if benefits are sustained at 12 months follow-up.

\section{GETTING UNDER THE SKIN: STREPTOCCOCUS PYOGENES IN TOXIC SHOCK}

doi:10.1136/archdischild-2012-302724.0049

A Norrby-Teglund. Karolinska Institute, Stockholm, Sweden

Streptococcus pyogenes can cause a variety of diseases in immunocompetent individuals, from pharyngotonsillitis to life-threatening invasive diseases like streptococcal toxic shock syndrome and rapidly progressing deep tissue infections, such as necrotizing fasciitis. Necrotizing fasciitis is often seen in combination with toxic shock, which further increases morbidity and mortality.

To gain insight into the pathogenesis of severe deep tissue infections, we have utilized a snap-frozen tissue biopsy material collected from patients with various soft tissue infections, including necrotizing fascitiis, myositis, and cellulitis caused by $S$. pyogenes. All patients had received intravenous clindamycin in combination with a $\beta$-lactam antibiotic at admission.

The studies revealed that severe soft tissue infections are characterized by massive bacterial load, presence of important streptococcal virulence factors including soluble M1-protein, the cysteine protease SpeB and superantigens, DNAses, and heavy infiltration of inflammatory cells and inflammatory mediators. Analyses of host-microbe interactions at the tissue site of infection have furthermore provided in vivo evidence for many of the immune evasion strategies previously described in vitro. Important bacterial resistance mechanisms at the tissue site include exploitation of human phagocytic cells as host cells thereby allowing persistence intracellularly, as well as protection against antimicrobial peptides by $\mathrm{SpeB}$ retained at the bacterial surface through GRAB-a2-macroglobulin complexes. It is clear that the pathogenesis of severe streptococcal tissue infections is multifactorial in nature. This complexity is important to consider in the design of novel therapeutic strategies, where IVIG represent one immunomodulatory therapy that should be evaluated further.

\section{LATE ONSET NEONATAL SEPSIS (LOS) IN VERY LOW BIRTH WEIGHT INFANTS: A MULTICENTRIC STUDY IN THE NEOCOSUR SOUTH AMERICAN NETWORK}

doi:10.1136/archdischild-2012-302724.0050

'MJ Escalante, 'JL Tapia, 'I D'Apremont, ' $2 \mathrm{~L}$ Villarroel, ${ }^{3} \mathrm{JM}$ Ceriani-Cernadas, ${ }^{4} \mathrm{~A}$ Bancalari. 'Neonatology; ${ }^{2}$ Department of Public Health, Pontifical Catholic University of Chile, Santiago, Chile; ${ }^{3}$ Hospital Italiano de Buenos Aires, Buenos Aires, Argentina, ${ }^{4}$ Hospital Guillermo Grant Benavente, Concepción, Chile

Background LOS is an important cause of mortality and morbidity among very low birth weight (VLBW) infants.

Aim To determine the incidence, bacteriology and associated morbidity to LOS over a 10 year period in a South American Network.

Methods Data were prospectively collected with predefined diagnostic criteria on all VLBW infants born in 18 centers from this Network, from 2001 through 2010. For numerical variables, mean and standard deviations were calculated. Students-t test or Chi Square tests were used for comparisons as appropriate. Logistic regression was used to assess association between sepsis and morbidity conditions.

Results 11651 VLBW were included, with a mean BW $1086 \pm 279 \mathrm{~g}$ and GA of $29.9 \pm 3$ weeks. A $19 \%$ acquired LOS, with a slight decrease in incidence from $19.5 \%$ in the $2001-2005$ period to $17.5 \%$ in 2010 . There was a wide intercenter variability from $5.9 \%$ to $29.6 \%$. The most common pathogens were CONS (53\%) and Staphylococcus aureus $(11 \%)$. Infants who developed LOS were significantly smaller by weight and gestational age. Multivariate logistic regression analysis showed a positive association between LOS and an increased risk for patent ductus arteriosus (OR: 1.510 [95\% CI: 1.113-2.049]), NEC (OR: 0.427 [95\% CI: 0.373-0.488]) and mechanical ventilation (OR: 0.383 [95\% CI: 0.327-0.449]).

Conclusions LOS remains an important cause of morbidity among VLBW infants with a wide intercenter variability. Decreasing LOS is a present important challenge for neonatal centers and networks may contribute in this purpose.

\section{RESISTIN - A NOVEL FEATURE IN THE DIAGNOSIS OF SEPSIS IN PREMATURE NEONATES}

doi:10.1136/archdischild-2012-302724.0051

'S Ozkiraz, ${ }^{2 Z}$ Gokmen, ${ }^{3} \mathrm{~S}$ Kulaksizoglu, ${ }^{3} \mathrm{H}$ Kilicdag, ${ }^{4} \mathrm{D}$ Ozel, ${ }^{3} \mathrm{~A}$ Ecevit, ${ }^{3} \mathrm{~A}$ Tarcan. ${ }^{1}$ Antalya Training and Research Hospital, Antalya; ${ }^{2}$ Konya Training and Research Hospital, Konya; ${ }^{3}$ Baskent University Hospital, Ankara; ${ }^{4} A$ kdeniz University, Antalya, Turkey

Objective To evaluate the efficacy of resistin in the diagnosis of sepsis and to compare with C-reactive protein (CRP) in preterm infants.

Study Design Totally 80 preterm infants were prospectively included in the study. Blood samples were collected within the first hour of life, on first and third days of sepsis for basal resistin, basal CRP, CRP-1, CRP-3, resistin-1 and resistin-3 levels. Septic patients were divided into two groups as Gr-negative and Gr-positive sepsis group.

Results Basal resistin and CRP levels were 14.0 (4.7-31.1) ng/ml and $0.5(0-23) \mathrm{mg} / \mathrm{dL}$. Culture-proven sepsis was diagnosed in 20 infants. Resistin-1 and resistin-3 were significantly higher than basal resistin levels $(p<0.01)$ and positively correlated with CRP. The area under curve values for CRP and resistin were 0.714 and 0.984 , respectively ( $p=0.039$ ). Resistin-1 and resistin-3 levels were significantly higher in Gr-negative sepsis group than Gr-positive $(\mathrm{p}<0.001)$.

Conclusion We showed that resistin had an efficacy superior to that of CRP in the diagnosis of sepsis in preterm infants. Resistin can be used as an early marker for sepsis in premature infants. Further studies are needed in larger groups to better understand the role of resistin to determine cut-off values for Gram-negative and positive sepsis.

52 PAEDIATRIC NEUROLOGICAL DISEASES: WHAT DOES ACTIVE CANADIAN SURVEILLANCE TELL US?

doi:10.1136/archdischild-2012-302724.0052 